\title{
Factors affecting exhaled nitric oxide measurements: the effect of
}

\section{sex}

\author{
D Robin Taylor*1, Piush Mandhane ${ }^{2}$, Justina M Greene ${ }^{2}$, Robert J Hancox ${ }^{3}$, \\ Sue Filsell ${ }^{3}$, Christene R McLachlan ${ }^{3}$, Avis J Williamson ${ }^{3}$, Jan O Cowan ${ }^{1}$, \\ Andrew D Smith ${ }^{1}$ and Malcolm R Sears ${ }^{2}$
}

\begin{abstract}
Address: ${ }^{1}$ Department of Respiratory Medicine, Dunedin School of Medicine, University of Otago, Dunedin, New Zealand, ${ }^{2}$ Firestone Institute for Respiratory Health, Department of Medicine, McMaster University, Hamilton, Ontario, Canada and ${ }^{3}$ Dunedin Multidisciplinary Health and Development Research Unit, Dunedin School of Medicine, University of Otago, Dunedin, New Zealand

Email: D Robin Taylor* - robin.taylor@stonebow.otago.ac.nz; Piush Mandhane - p.mandhane@utoronto.ca; Justina M Greene - justina.greene@utoronto.ca; Robert J Hancox - bob.hancox@otago.ac.nz; Sue Filsell - SueF@healthotago.co.nz; Christene R McLachlan - chris.mclachlan@otagodhb.govt.nz; Avis J Williamson - resp.ed@otago.ac.nz; Jan O Cowan - jan.cowan@stonebow.otago.ac.nz; Andrew D Smith - adsmith2000@ntlworld.com; Malcolm R Sears - jmsears@interlynx.net

* Corresponding author
\end{abstract}

Published: 15 November 2007

Respiratory Research 2007, 8:82 doi:10.1/86/1465-992I-8-82
Received: 2 June 2007

Accepted: 15 November 2007

This article is available from: http://respiratory-research.com/content/8/I/82

(c) 2007 Taylor et al; licensee BioMed Central Ltd.

This is an Open Access article distributed under the terms of the Creative Commons Attribution License (http://creativecommons.org/licenses/by/2.0), which permits unrestricted use, distribution, and reproduction in any medium, provided the original work is properly cited.

\begin{abstract}
Background: Exhaled nitric oxide $\left(\mathrm{F}_{\mathrm{E}} \mathrm{NO}\right)$ measurements are used as a surrogate marker for eosinophilic airway inflammation. However, many constitutional and environmental factors affect $\mathrm{F}_{\mathrm{E}} \mathrm{NO}$, making it difficult to devise reference values. Our aim was to evaluate the relative importance of factors affecting $\mathrm{F}_{\mathrm{E}} \mathrm{NO}$ in a well characterised adult population.

Methods: Data were obtained from 895 members of the Dunedin Multidisciplinary Health and Development Study at age 32. The effects of sex, height, weight, lung function indices, smoking, atopy, asthma and rhinitis on $\mathrm{F}_{\mathrm{E}} \mathrm{NO}$ were explored by unadjusted and adjusted linear regression analyses.

Results: The effect of sex on $\mathrm{F}_{\mathrm{E}} \mathrm{NO}$ was both statistically and clinically significant, with $\mathrm{F}_{\mathrm{E}} \mathrm{NO}$ levels approximately $25 \%$ less in females. Overall, current smoking reduced $\mathrm{F}_{\mathrm{E}} \mathrm{NO}$ up to $50 \%$, but this effect occurred predominantly in those who smoked on the day of the $\mathrm{F}_{\mathrm{E}} \mathrm{NO}$ measurement. Atopy increased $\mathrm{F}_{\mathrm{E}} \mathrm{NO}$ by $60 \%$. The sex-related differences in $\mathrm{F}_{\mathrm{E}} \mathrm{NO}$ remained significant $(\mathrm{p}<0.00 \mathrm{I})$ after controlling for all other significant factors affecting $\mathrm{F}_{\mathrm{E}} \mathrm{NO}$.

Conclusion: Even after adjustment, $\mathrm{F}_{\mathrm{E}} \mathrm{NO}$ values are significantly different in males and females. The derivation of reference values and the interpretation of $\mathrm{F}_{\mathrm{E}} \mathrm{NO}$ in the clinical setting should be stratified by sex. Other common factors such as current smoking and atopy also require to be taken into account.
\end{abstract}

\section{Background}

Measurement of exhaled nitric oxide $\left(\mathrm{F}_{\mathrm{E}} \mathrm{NO}\right)$ is increasingly recognised as an important addition to pulmonary function testing in clinical practice [1]. $\mathrm{F}_{\mathrm{E}} \mathrm{NO}$ may be used as a surrogate marker for airway eosinophilia [2], and as an alternative to other more invasive or time-consuming 
assessments of airway pathology such as induced sputum, [2,3] bronchial lavage fluid, [4] or mucosal biopsy. [5-7] Against this background, $\mathrm{F}_{\mathrm{E}} \mathrm{NO}$ measurements are increasingly being used to clarify the aetiology of non-specific respiratory symptoms as well as monitor levels of inflammation in conditions characterised by airway eosinophilia [8].

There are a number of demographic and biological factors which cause variation in $\mathrm{F}_{\mathrm{E}} \mathrm{NO}$ levels. The commonest are cigarette smoking $[9,10]$ and atopy [11-13] with or without allergic rhinitis. [13,14] Others include age $[15,16]$, and IgE levels [17]. However, conflicting results concerning the importance of these factors has precluded a clear definition of so-called "normal" values. Buchvald et al. have reported reference values in a large population of children, but important biological confounders were evaluated only by questionnaire. [15] The same issues were addressed more recently in adults by Olin et al. [18] and Travers et al. [19], and Travers et al. provided reference ranges which sought to take account of commonly encountered variables which affect $\mathrm{F}_{\mathrm{E}} \mathrm{NO}$. However, there are some significant inconsistencies between these reports, not least in respect of the effects of sex on $\mathrm{F}_{\mathrm{E}} \mathrm{NO}$. Clearly further data are needed so that routine $\mathrm{F}_{\mathrm{E}} \mathrm{NO}$ measurements can be interpreted appropriately. In the present study, comprising a well characterised cohort of nearly 1000 32-year old individuals born in Dunedin, New Zealand, we obtained detailed clinical and laboratory information regarding factors affecting $\mathrm{F}_{\mathrm{E}} \mathrm{NO}$, and their potential relevance to reference ranges for $\mathrm{F}_{\mathrm{E}} \mathrm{NO}$ was evaluated.

\section{Methods}

The Dunedin Multidisciplinary Health and Development Study is a cohort study of 1037 children (52\% male) born between April 1972 and March 1973. [20,21] Follow-up assessments have been conducted at ages 3, 5, 7, 9, 11, 13, $15,18,21,26$, and at 32 years, at which time 972 (96\%) of 1015 living Study members participated.

At age 32, Study members were questioned about current and previous asthma, as well as symptoms of wheezing, cough, episodic shortness of breath, hay fever and rhinitis. Current asthma was defined as reported diagnosed asthma with symptoms in the last 12 months. Current wheezing was recorded as any wheeze in the last 12 months but excluding subjects with only one or two episodes each lasting less than 1 hour. Asthma treatment was any inhaled bronchodilator, corticosteroid or cromoglycate medication. Current smoking was defined as smoking tobacco cigarettes daily for at least one month during the previous 12 months, or smoking cannabis 6 or more times during the previous 12 months. Current smokers were further subdivided into two groups: those who did and did not smoke on the study day. Ex-smokers were defined as having discontinued for at least 12 months.

Height and weight in light clothing without shoes were measured to calculate body mass index (BMI) in $\mathrm{kg} / \mathrm{m}^{2}$. $\mathrm{F}_{\mathrm{E}} \mathrm{NO}$ was then measured on-line using a Logan LR 2000 series chemiluminescence analyser (Logan Research Ltd., Rochester, England) in accordance with ATS/ERS guidelines at a flow rate of $50 \mathrm{~mL} / \mathrm{sec}$. [22] Exhaled nitric oxide in parts per billion ( $\mathrm{ppb}$ ) was recorded continuously throughout expiration. Individual results were read at the first nitric oxide plateau and the mean of two acceptable tests was recorded. A third was obtained only where one or both of the first two were considered to be technically unsatisfactory. The NO recording was determined for each test by two observers on a separate occasion. The first 44 Study members were tested using a flow rate of $250 \mathrm{~mL} /$ second and results were adjusted to $50 \mathrm{~mL} /$ second using a previously validated formula [23].

$\mathrm{F}_{\mathrm{E}} \mathrm{NO}$ measurements were obtained immediately prior to carrying out spirometry. Skin prick testing included house dust mite (D. pteronyssinus), grass, cat, dog, horse, cockroach, wool, Aspergillus fumigatus, alternaria, penicillium, and cladosporium. A weal diameter $3 \mathrm{~mm}$ or greater than the saline control was considered positive. Atopy was defined as a positive response to one or more allergens. A blood sample was obtained for eosinophil count and total serum immunoglobulin E (IgE).

\section{Statistical analysis}

Study members who were pregnant at the time of assessment $(n=31)$ were excluded from all analyses. $\mathrm{F}_{\mathrm{E}} \mathrm{NO}$ measurements were not normally distributed, and were log transformed prior to analysis. Both univariate and multivariate linear regression analyses were performed to identify those factors which significantly affected $\mathrm{F}_{\mathrm{E}} \mathrm{NO}$ levels and to derive regression equations, with stratification for those factors which were shown to significantly affect exhaled nitric oxide levels. The selection of appropriate linear regression models was based on maximum Rsquare and an examination of the residuals, to ensure an adequate model fit. Significant interaction terms ( $\mathrm{p}<$ $0.05)$ were retained in the model. Results are presented as anti-log values with 95\% confidence intervals following back-transformation.

\section{Ethics}

The Otago Ethics Committee approved the study and written informed consent was obtained.

\section{Results}

Eight hundred and ninety-five Study members completed the respiratory procedures in the Study. Of these, 471 (52.6\%) were male, 486 (54.8\%) were atopic, 349 
(39.0\%) had rhinitis/hay fever, 253 (28.3\%) had current wheeze, 156 (17.4\%) had current asthma, and 54 (6.6\%) were using inhaled corticosteroid treatment. Three hundred and ninety five were current smokers (44.1\%), of whom 235 (59.5\%) smoked on the study assessment day prior to testing, and 107 were ex-smokers (12.0\%). Two hundred and fifteen were cannabis smokers (24\%), of whom $78(8.7 \%)$ smoked cannabis alone.
The $\mathrm{F}_{\mathrm{E}} \mathrm{NO}$ values obtained from the Study population are shown in Table 1. Data relating to smoking status are shown in Table 2. $\mathrm{F}_{\mathrm{E}} \mathrm{NO}$ was on average $25 \%$ higher in males than females (males: 15.3 ppb [95\% C.I.:14.3-16.3] versus females: $11.6 \mathrm{ppb}$ [95\% C.I.: 11.0-12.4]; $\mathrm{p}<0.0001$; Table 1). Unadjusted analyses revealed that, for all Study members, there were also significant differences in $\mathrm{F}_{\mathrm{E}} \mathrm{NO}$ in relation to height, $\mathrm{FEV}_{1}$, $\mathrm{FEV}_{1} \%$ predicted and FVC (Table 3 ). However, none of

Table I: Mean values (with $95 \%$ confidence intervals) for $\mathrm{F}_{\mathrm{E}} \mathrm{NO}$ stratified by sex, smoking, atopy, rhinitis current wheeze and asthma. * not all Study members underwent skin testing

\begin{tabular}{|c|c|c|c|c|c|c|c|c|c|c|c|c|}
\hline All & \multicolumn{12}{|c|}{$\begin{array}{c}\text { All subjects, } n=895 \\
13.4(12.9,14.1)\end{array}$} \\
\hline Gender & \multicolumn{6}{|c|}{$\begin{array}{l}\text { Male, } n=47 \mid \\
15.3(\mid 4.3,16.3)\end{array}$} & \multicolumn{6}{|c|}{$\begin{array}{l}\text { Female, } n=424 \\
\text { II.6 (I I.0, I } 2.4)\end{array}$} \\
\hline \multirow[t]{2}{*}{ Smoking } & \multicolumn{2}{|c|}{$\begin{array}{c}\text { Current smoker } \\
\text { (smoked on the day of } \\
\text { testing) }\end{array}$} & \multicolumn{2}{|c|}{$\begin{array}{l}\text { Current smoker (not } \\
\text { smoked on the day of } \\
\text { testing) }\end{array}$} & \multicolumn{2}{|c|}{$\begin{array}{l}\text { Ex-smoker or non- } \\
\text { smoker }\end{array}$} & \multicolumn{2}{|c|}{$\begin{array}{c}\text { Current smoker } \\
\text { (smoked on the day of } \\
\text { testing) }\end{array}$} & \multicolumn{2}{|c|}{$\begin{array}{l}\text { Current smoker (not } \\
\text { smoked on the day of } \\
\text { testing) }\end{array}$} & \multicolumn{2}{|c|}{$\begin{array}{c}\text { Ex-smoker or non } \\
\text { smoker }\end{array}$} \\
\hline & \multicolumn{2}{|c|}{$\begin{array}{c}\mathrm{n}=133 \\
8.8 \\
(7.9,9.7)\end{array}$} & \multicolumn{2}{|c|}{$\begin{array}{c}n=102 \\
16.6 \\
(14.7,18.9)\end{array}$} & \multicolumn{2}{|c|}{$\begin{array}{c}n=236 \\
20.2 \\
(18.6,21.9)\end{array}$} & \multicolumn{2}{|c|}{$\begin{array}{c}n=102 \\
7.4 \\
(6.7,8.2)\end{array}$} & \multicolumn{2}{|c|}{$\begin{array}{c}n=58 \\
12.3 \\
(10.2,14.7)\end{array}$} & \multicolumn{2}{|c|}{$\begin{array}{c}n=264 \\
13.7 \\
(12.8,14.7)\end{array}$} \\
\hline \multirow[t]{2}{*}{ Atopy } & Yes & No & Yes & No & Yes & No & Yes & No & Yes & No & Yes & No \\
\hline & $\begin{array}{c}n=68 \\
9.9 \\
(8.6,11.5)\end{array}$ & $\begin{array}{c}n=62 \\
7.6 \\
(6.7,8.7)\end{array}$ & $\begin{array}{c}n=61 \\
19.8 \\
(16.6,23.6)\end{array}$ & $\begin{array}{c}n=41 \\
12.9 \\
(I 1 . I, 14.9)\end{array}$ & $\begin{array}{c}n=|4| \\
24.8 \\
(22.2,27.7)\end{array}$ & $\begin{array}{c}n=92 \\
14.9 \\
(13.6,16.3)\end{array}$ & $\begin{array}{c}n=32 \\
9.5 \\
(7.8,11.5)\end{array}$ & $\begin{array}{c}n=69 \\
6.7 \\
(5.9,7.5)\end{array}$ & $\begin{array}{c}n=35 \\
14.0 \\
(10.6,18.5)\end{array}$ & $\begin{array}{c}n=23 \\
10.0 \\
(8.2,12.2)\end{array}$ & $\begin{array}{c}n=149 \\
15.9 \\
(14.4,17.6)\end{array}$ & $\begin{array}{c}n 114 \\
11.2 \\
(10.3,12.2)\end{array}$ \\
\hline \multirow[t]{2}{*}{ Rhinitis } & Yes & No & Yes & No & Yes & No & Yes & No & Yes & No & Yes & No \\
\hline & $\begin{array}{c}n=50 \\
9.2 \\
(8.0,10.7)\end{array}$ & $\begin{array}{c}n=83 \\
8.5 \\
(7.4,9.7)\end{array}$ & $\begin{array}{c}n=30 \\
21.0 \\
(16.0,27.5)\end{array}$ & $\begin{array}{c}n=72 \\
15.1 \\
(13.2,17.4)\end{array}$ & $\begin{array}{c}n=97 \\
24.0 \\
(21.2,27.2)\end{array}$ & $\begin{array}{c}n=139 \\
17.9 \\
(16.1,19.9)\end{array}$ & $\begin{array}{c}n=33 \\
9.0 \\
(7.5,10.9)\end{array}$ & $\begin{array}{c}n=69 \\
6.8 \\
(6.0,7.7)\end{array}$ & $\begin{array}{c}n=23 \\
13.4 \\
(10.1,17.8)\end{array}$ & $\begin{array}{c}n=35 \\
11.6 \\
(9.0,14.9)\end{array}$ & $\begin{array}{c}n=116 \\
16.1 \\
(14.3,18.0)\end{array}$ & $\begin{array}{c}n=148 \\
12.1 \\
(11.1,13.1)\end{array}$ \\
\hline $\begin{array}{l}\text { Current } \\
\text { wheeze }\end{array}$ & Yes & No & Yes & No & Yes & No & Yes & No & Yes & No & Yes & No \\
\hline All & $\begin{array}{c}n=49 \\
8.4 \\
(6.9,10.2)\end{array}$ & $\begin{array}{c}n=84 \\
9.0 \\
(8.0,10.0)\end{array}$ & $\begin{array}{c}n=35 \\
17.7 \\
(13.4,23.3)\end{array}$ & $\begin{array}{c}n=67 \\
16.1 \\
(14.1,18.5)\end{array}$ & $\begin{array}{c}n=53 \\
28.4 \\
(23.0,34.9)\end{array}$ & $\begin{array}{c}n=183 \\
18.3 \\
(16.9,19.9)\end{array}$ & $\begin{array}{c}n=41 \\
7.6 \\
(6.4,9.1)\end{array}$ & $\begin{array}{c}n=61 \\
7.3 \\
(6.4,8.4)\end{array}$ & $\begin{array}{c}n=18 \\
15.2 \\
(9.4,24.3)\end{array}$ & $\begin{array}{c}n=40 \\
I I . I \\
(9.3, I 3.3)\end{array}$ & $\begin{array}{c}n=57 \\
16.8 \\
(14.2,19.8)\end{array}$ & $\begin{array}{c}n=207 \\
12.9 \\
(12.0,13.9)\end{array}$ \\
\hline $\begin{array}{l}\text { Taking } \\
\text { ICS }\end{array}$ & $\begin{array}{c}n=8 \\
8.0 \\
(4.1,15.6)\end{array}$ & Nil & $\begin{array}{c}n=5 \\
24.0 \\
(12.7,45.5)\end{array}$ & $\begin{array}{l}n=1 \\
72.9\end{array}$ & $\begin{array}{c}n=17 \\
27.5 \\
(19.6,38.7)\end{array}$ & $\begin{array}{l}n=2 \\
28.5 \\
(\mathrm{NA})\end{array}$ & $\begin{array}{c}n=3 \\
8.8 \\
(2.0,40.0)\end{array}$ & $\begin{array}{c}n=4 \\
9.4 \\
(1.9,45.2)\end{array}$ & $\begin{array}{c}n=3 \\
32.4 \\
(9.3,112.0)\end{array}$ & Nil & $\begin{array}{c}n=13 \\
17.3 \\
(13.1,22.8)\end{array}$ & $\begin{array}{c}n=3 \\
17.8 \\
(3.3,96.9)\end{array}$ \\
\hline $\begin{array}{l}\text { Not } \\
\text { taking } \\
\text { ICS }\end{array}$ & $\begin{array}{c}n=4 I \\
8.5 \\
(6.9,10.4)\end{array}$ & $\begin{array}{c}n=84 \\
9.0 \\
(8.0,10.0)\end{array}$ & $\begin{array}{c}n=30 \\
12.3 \\
(16.8,22.9)\end{array}$ & $\begin{array}{c}n=66 \\
15.8 \\
(13.8,17.9)\end{array}$ & $\begin{array}{c}n=36 \\
28.8 \\
(21.9,37.8)\end{array}$ & $\begin{array}{c}n=|8| \\
18.2 \\
(16.8,19.8)\end{array}$ & $\begin{array}{c}n=38 \\
7.5 \\
(6.2,9.1)\end{array}$ & $\begin{array}{c}n=57 \\
7.2 \\
(6.4,8.2)\end{array}$ & $\begin{array}{c}n=15 \\
13.0 \\
(7.7,22.1)\end{array}$ & $\begin{array}{c}n=40 \\
I I .1 \\
(9.3,13.1)\end{array}$ & $\begin{array}{c}n=44 \\
16.7 \\
(13.6,20.5)\end{array}$ & $\begin{array}{c}n=204 \\
12.9 \\
(11.9,13.9)\end{array}$ \\
\hline $\begin{array}{l}\text { Current } \\
\text { asthma }\end{array}$ & Yes & No & Yes & No & Yes & No & Yes & No & Yes & No & Yes & No \\
\hline All & $\begin{array}{c}n=21 \\
9.8 \\
(6.9,14.0)\end{array}$ & $\begin{array}{c}n=112 \\
8.6 \\
(7.7,9.5)\end{array}$ & $\begin{array}{c}n=27 \\
21.7 \\
(16.5,28.5)\end{array}$ & $\begin{array}{c}n=75 \\
15.1 \\
(13.2,17.4)\end{array}$ & $\begin{array}{c}n=39 \\
29.7 \\
(23.7,37.2)\end{array}$ & $\begin{array}{c}n=197 \\
18.7 \\
(17.2,20.4)\end{array}$ & $\begin{array}{c}n=18 \\
9.5 \\
(6.5,13.9)\end{array}$ & $\begin{array}{c}n=84 \\
7.0 \\
(6.4,7.8)\end{array}$ & $\begin{array}{c}n=10 \\
28.0 \\
(14.7,53.3)\end{array}$ & $\begin{array}{c}n=48 \\
10.3 \\
(8.9,12.0)\end{array}$ & $\begin{array}{c}n=41 \\
15.7 \\
(12.7,19.5)\end{array}$ & $\begin{array}{c}n=223 \\
13.3 \\
(12.4,14.3)\end{array}$ \\
\hline $\begin{array}{l}\text { Taking } \\
\text { ICS }\end{array}$ & $\begin{array}{c}n=8 \\
8.0 \\
(4.1,15.6)\end{array}$ & Nil & $\begin{array}{c}n=6 \\
28.9 \\
(14.7,57.0)\end{array}$ & Nil & $\begin{array}{c}n=18 \\
26.5 \\
(19.1,36.8)\end{array}$ & $\begin{array}{l}n=1 \\
58.3\end{array}$ & $\begin{array}{c}n=5 \\
I I .0 \\
(3.8,32.2)\end{array}$ & $\begin{array}{c}n=2 \\
5.7 \\
(0.5,72.1)\end{array}$ & $\begin{array}{c}n=3 \\
32.4 \\
(9.3,112.0)\end{array}$ & Nil & $\begin{array}{c}n=14 \\
18.3 \\
(13.8,24.3)\end{array}$ & $\begin{array}{c}n=2 \\
12.0 \\
(3.1,47.4)\end{array}$ \\
\hline $\begin{array}{l}\text { Not } \\
\text { taking } \\
\text { ICS }\end{array}$ & $\begin{array}{c}n=13 \\
11.2 \\
(7.1,17.8)\end{array}$ & $\begin{array}{c}n=112 \\
8.6 \\
(7.7,9.5)\end{array}$ & $\begin{array}{c}n=21 \\
20.0 \\
(14.6,27.4)\end{array}$ & $\begin{array}{c}n=75 \\
15.1 \\
(13.2,17.4)\end{array}$ & $\begin{array}{c}n=21 \\
32.7 \\
(23.5,45.6)\end{array}$ & $\begin{array}{c}n=196 \\
18.6 \\
(17.1,20.2)\end{array}$ & $\begin{array}{c}n=13 \\
9.0 \\
(5.8,14.2)\end{array}$ & $\begin{array}{c}n=82 \\
7.1 \\
(6.4,7.8)\end{array}$ & $\begin{array}{c}n=7 \\
26.4 \\
(9.9,69.9)\end{array}$ & $\begin{array}{c}n=48 \\
10.3 \\
(8.9,12.0)\end{array}$ & $\begin{array}{c}n=27 \\
14.6 \\
(10.8,19.5)\end{array}$ & $\begin{array}{c}n=221 \\
13.3 \\
(12.4,14.4)\end{array}$ \\
\hline
\end{tabular}


Table 2: Mean values (with $95 \%$ confidence intervals) for $F_{E}$ NO, stratified by smoking status and sex

\begin{tabular}{|c|c|c|c|}
\hline$F_{E} N O(p p b)$ Mean $95 \%$ C.I. & All subjects & Males & Females \\
\hline All Study members & $\begin{array}{c}n=895 \\
13.4 \\
(12.8,14.1)\end{array}$ & $\begin{array}{c}n=47 \mid \\
15.3 \\
(14.3,16.3)\end{array}$ & $\begin{array}{l}n=424 \\
11.6 \\
(11.0,12.4)\end{array}$ \\
\hline All current smokers (within last I 2 months) & $\begin{array}{l}n=395 \\
10.4 \\
(9.7,11.1)\end{array}$ & $\begin{array}{c}n=235 \\
\quad 11.6 \\
(10.6,12.6)\end{array}$ & $\begin{array}{l}\mathrm{n}=160 \\
8.9 \\
(8.1,9.8)\end{array}$ \\
\hline $\begin{array}{l}\text { Current smokers who smoked on the day of } \\
F_{E} \text { NO testing }\end{array}$ & $\begin{array}{l}n=235 \\
8.2 \\
(7.6,8.8)\end{array}$ & $\begin{array}{l}\mathrm{n}=133 \\
8.8 \\
(7.9,9.7)\end{array}$ & $\begin{array}{l}n=102 \\
7.4 \\
(6.7,8.2)\end{array}$ \\
\hline $\begin{array}{l}\text { Current smokers who did not smoke on day of } \\
F_{\mathrm{E}} \text { NO testing }\end{array}$ & $\begin{array}{c}n=160 \\
14.9 \\
(13.4,16.6)\end{array}$ & $\begin{array}{l}n=102 \\
16.6 \\
(14.7,18.9)\end{array}$ & $\begin{array}{c}n=58 \\
12.3 \\
(10.2,14.7)\end{array}$ \\
\hline Ex-smokers (greater than 12 months) & $\begin{array}{c}n=107 \\
16.0 \\
(14.3,17.8)\end{array}$ & $\begin{array}{c}n=36 \\
21.1 \\
(17.6,25.3)\end{array}$ & $\begin{array}{c}n=7 \mid \\
13.8 \\
(12.2,15.7)\end{array}$ \\
\hline Never smokers & $\begin{array}{c}n=393 \\
16.6 \\
(15.6,17.7)\end{array}$ & $\begin{array}{c}n=200 \\
20.0 \\
(18.3,22.0)\end{array}$ & $\begin{array}{c}n=193 \\
13.6 \\
(12.5,14.8)\end{array}$ \\
\hline
\end{tabular}

these factors remained significant after stratifying by sex. In contrast, current smoking (on the day of testing), atopy, $\log$ IgE, history of rhinitis, and current asthma and the use of inhaled corticosteroids remained significant in both males and females.

In the adjusted regression analyses, significant predictors of FENO were sex, body mass index (BMI), current smoking (on the day of testing), atopy, current asthma, and the interaction between sex and smoking. Current wheeze was not a significant factor. This was perhaps because of the significant confounding relationship between current smoking (resulting in reduced FENO) and wheeze ( $\mathrm{p}=$ 0003; model 4; see Additional File 1). Details of all the models examined are provided in Additional File 1.

After controlling for all of the significant factors affecting $\mathrm{F}_{\mathrm{E}} \mathrm{NO}$, the sex-related differences in $\mathrm{F}_{\mathrm{E}} \mathrm{NO}$ remained significant $(\mathrm{p}<0.001)$. The factors which significantly affected $\mathrm{F}_{\mathrm{E}} \mathrm{NO}$ were different for males and females. For males, current smoking (all), current asthma, and atopy (any positive SPT $\geq 3 \mathrm{~mm}$ over the negative control) were significant independent predictors of $\mathrm{F}_{\mathrm{E}} \mathrm{NO}$. For females, while current smoking (all), current asthma, and atopy

Table 3: Factors affecting $F_{E} N O$ by linear regression analysis, without controlling for any other factors Magnitude of effect $=$ change compared to reference group (females, non-smokers, non-atopics, non-rhinitics, non-wheezers, or non-asthmatics)

\begin{tabular}{|c|c|c|c|c|c|c|}
\hline \multirow[b]{2}{*}{ Factor } & \multicolumn{2}{|c|}{ All } & \multicolumn{2}{|c|}{ Males } & \multicolumn{2}{|c|}{ Females } \\
\hline & $\begin{array}{l}\text { Magnitude of } \\
\text { effect* }^{*}\end{array}$ & Significance & $\begin{array}{l}\text { Magnitude of } \\
\text { effect* }\end{array}$ & Significance & $\begin{array}{l}\text { Magnitude of } \\
\text { effect* }\end{array}$ & Significance \\
\hline Female Gender & 0.7605 & $<0.0001$ & \multicolumn{2}{|c|}{--- } & \multicolumn{2}{|c|}{--- } \\
\hline BMI & 1.0015 & 0.7571 & 1.0045 & 0.5600 & 0.9966 & 0.5503 \\
\hline Height & 1.0013 & $<0.0001$ & 1.0003 & 0.5859 & 1.0007 & 0.0969 \\
\hline FEV & 1.1315 & $<0.0001$ & 0.9956 & 0.9328 & 1.0229 & 0.7230 \\
\hline FEV, \% predicted & 0.9957 & 0.0166 & 0.9988 & 0.6422 & 0.9981 & 0.4432 \\
\hline FVC & I. 1058 & $<0.0001$ & 0.9832 & 0.6963 & 1.0443 & 0.4044 \\
\hline $\begin{array}{l}\text { Current smoker } \\
\text { (smoked on day of testing) }\end{array}$ & 0.49600 & $<0.0001$ & 0.43350 & $<0.0001$ & 0.54337 & $<0.0001$ \\
\hline $\begin{array}{l}\text { Current smoker (within last I } 2 \\
\text { months, not smoked on day of } \\
\text { testing) }\end{array}$ & 0.90579 & 0.0825 & 0.82377 & 0.0091 & 0.89582 & 0.1945 \\
\hline Atopy ( $\geq 3 \mathrm{~mm}$ ) & 1.6028 & $<0.0001$ & 1.60363 & $<0.0001$ & 1.5472 & $<0.0001$ \\
\hline Log IgE & I.3897 & $<0.0001$ & I.397| & $<0.0001$ & 1.3235 & $<0.0001$ \\
\hline Current rhinitis & 1.3126 & $<0.0001$ & 1.2868 & 0.0002 & 1.3670 & $<0.0001$ \\
\hline Current wheeze & 1.1009 & 0.0617 & 1.0906 & 0.2349 & 1.1004 & 0.1658 \\
\hline Current asthma & 1.4132 & $<0.0001$ & 1.4434 & $<0.0001$ & 1.3495 & 0.0003 \\
\hline Using ICS & 1.3904 & 0.0004 & 1.3780 & 0.0134 & 1.3755 & 0.0130 \\
\hline
\end{tabular}


(any positive SPT $\geq 3 \mathrm{~mm}$ over the negative control) were significant independent predictors of $\mathrm{F}_{\mathrm{E}} \mathrm{NO}$, those females who were current smokers and also had asthma had an additional increase in their $\mathrm{F}_{\mathrm{E}} \mathrm{NO}\left(\mathrm{F}_{\mathrm{E}} \mathrm{NO}\right.$ increased by 131\%; $\mathrm{p}=0.001$; Table 4).

Based on these results, the equations for predicting $\mathrm{F}_{\mathrm{E}} \mathrm{NO}$ in our study cohort were:

For males: $\log \mathrm{F}_{\mathrm{E}} \mathrm{NO}=1.1932-0.3496^{*}$ current smoking (smoked day of testing) - 0.0940* current smoking (not smoked day of testing) $+0.16511^{*}$ atopy + $0.0973 *$ asthma

$\left(\mathrm{R}^{2}=0.3434\right)$

For females: $\log \mathrm{F}_{\mathrm{E}} \mathrm{NO}=1.0533-0.2407^{*}$ current smoking (smoked day of testing) - 0.1160* current smoking (not smoked on day of testing) $+0.0388^{*}$ asthma + $0.1355^{*}$ atopy $+0.0531 *$ current smoking (smoked day of testing)*asthma $+0.3630^{*}$ current smoking (not smoked day of testing)* asthma

$\left(\mathrm{R}^{2}=0.2760\right)$

where, for the terms current smoking, atopy and asthma, yes $=1$, and no $=0$.
Using these equations, the predicted values and ranges (95\% C.I.) for clinically important populations are presented in Table 5. For comparison, the actual values obtained from each subgroup of the study population are also presented.

\section{Discussion}

The results of the present study provide further evidence that sex is a major factor determining exhaled nitric oxide $\left(\mathrm{F}_{\mathrm{E}} \mathrm{NO}\right)$ measurements. Without adjusting for other factors such as atopy, current smoking, and diagnosed asthma, the mean $\mathrm{F}_{\mathrm{E}} \mathrm{NO}$ levels in males were significantly higher than in females $(\mathrm{p}=0.0001)$. However, even after appropriate adjustments, this difference persisted. The magnitude of the difference was approximately $25 \%$. This is clinically as well as statistically significant [24].

A review of the literature provides somewhat conflicting data regarding this issue. It is important to take account of the different methodologies used for $\mathrm{F}_{\mathrm{E}} \mathrm{NO}$ measurements when making comparisons between studies, particularly with regard to expiratory flow rates. However, within studies, significant differences between males and females will still be valid, and the balance of evidence suggests that sex-related differences in $\mathrm{F}_{\mathrm{E}} \mathrm{NO}$ are indeed important. In early investigations, both Jilma et al. [25] and Tsang et al. [26] reported sex-related differences in $\mathrm{F}_{\mathrm{E}} \mathrm{NO}$ whose magnitude $50 \%$ and $53 \%$ higher in males compared to females, respectively) was comparable to the present

Table 4: Adjusted linear regression models with $\mathrm{F}_{\mathrm{E}} \mathrm{NO}$ as the dependent variable

\begin{tabular}{|c|c|c|c|c|}
\hline Sample & Variables & Anti-log B-coefficient & p-value & R-square \\
\hline \multirow[t]{8}{*}{ All Study Members } & Intercept & 15.9 & $<0.0001$ & 0.331 \\
\hline & Sex & 0.69 & $<0.0001$ & \\
\hline & Current Smoking - smoked on the testing day & 0.45 & $<0.0001$ & \\
\hline & Current Smoking - not smoked on the testing day & 0.80 & 0.0014 & \\
\hline & Current Asthma & 1.26 & $<0.0001$ & \\
\hline & Atopy & 1.41 & $<0.0001$ & \\
\hline & Sex*Current Smoking - smoked on the testing day & 1.32 & 0.0025 & \\
\hline & Sex*Current Smoking - not smoked on the testing day & 1.10 & 0.3970 & \\
\hline \multirow[t]{5}{*}{ Males } & Intercept & 15.60 & $<0.0001$ & 0.343 \\
\hline & Current Smoking - smoked on the testing day & 0.45 & $<0.0001$ & \\
\hline & Current Smoking - not smoked on the testing day & 0.81 & 0.0021 & \\
\hline & Current Asthma & 1.25 & 0.0025 & \\
\hline & Atopy & 1.45 & $<0.0001$ & \\
\hline \multirow[t]{7}{*}{ Females } & Intercept & $\mid 1.31$ & $<0.0001$ & 0.276 \\
\hline & Current Smoking - smoked on the testing day & 0.57 & $<0.0001$ & \\
\hline & Current Smoking - not smoked on the testing day & 0.77 & 0.0022 & \\
\hline & Current Asthma & 1.09 & 0.3460 & \\
\hline & Atopy & 1.37 & $<0.0001$ & \\
\hline & $\begin{array}{l}\text { Current Asthma*Current Smoking - smoked on the testing } \\
\text { day }\end{array}$ & 1.13 & 0.4722 & \\
\hline & $\begin{array}{l}\text { Current Asthma*Current Smoking - not smoked on the } \\
\text { testing day }\end{array}$ & 2.31 & $<0.0001$ & \\
\hline
\end{tabular}


Table 5: Mean values and reference ranges for $F_{E} N O$ (with $95 \%$ confidence intervals), based on prediction equations for males and females. For comparison, the measured values (with $95 \%$ confidence intervals) obtained in the Study members are provided

\begin{tabular}{|c|c|c|c|c|c|}
\hline \multirow[b]{2}{*}{ Population } & & \multicolumn{2}{|c|}{ Males } & \multicolumn{2}{|c|}{ Females } \\
\hline & & $\mathrm{F}_{\mathrm{E}} \mathrm{NO}(\mathbf{p p b})$ & 95\% C.I. & $F_{E} N O(p p b)$ & 95\% C.I. \\
\hline \multirow[t]{2}{*}{ Non-smokers, non-atopic, non-asthmatic } & Predicted & 15.6 & $14.1,17.2$ & 11.3 & $10.3,12.4$ \\
\hline & Actual & 14.7 & $13.4,16.1$ & 11.2 & $10.3,12.2$ \\
\hline \multirow[t]{2}{*}{ Non-smokers, atopic, non-asthmatic } & Predicted & 22.6 & $18.3,28.0$ & 15.4 & $12.6,18.9$ \\
\hline & Actual & 23.1 & $20.4,26.2$ & 15.6 & $13.9,17.4$ \\
\hline \multirow[t]{2}{*}{ Non-smokers, non-atopic, asthmatic } & Predicted & 19.5 & $15.3,24.9$ & 12.4 & $9.4,16.3$ \\
\hline & Actual & 22.5 & $8.0,63.8$ & 11.5 & $7.0,18.9$ \\
\hline \multirow[t]{2}{*}{ Non-smokers, atopic, asthmatic } & Predicted & 28.3 & $19.8,40.5$ & 16.9 & $11.5,24.9$ \\
\hline & Actual & 30.4 & $23.9,38.7$ & 17.2 & $13.5,22.1$ \\
\hline \multirow{2}{*}{$\begin{array}{l}\text { Smokers (not smoked on the day of } \\
\text { testing), non-atopic, non-asthmatic }\end{array}$} & Predicted & 12.6 & $9.9,18.2$ & 8.7 & $6.7,11.3$ \\
\hline & Actual & 13.0 & $11.0,15.2$ & 9.6 & $7.8,11.7$ \\
\hline \multirow{2}{*}{$\begin{array}{l}\text { Smokers (not smoked on the day of } \\
\text { testing), atopic, non-asthmatic }\end{array}$} & Predicted & 18.2 & $12.8,25.5$ & 11.8 & $8.2,17.2$ \\
\hline & Actual & 17.6 & |4.|, 22.0 & 10.9 & $8.7,13.8$ \\
\hline \multirow{2}{*}{$\begin{array}{l}\text { Smokers (not smoked on the day of } \\
\text { testing), non-atopic, asthmatic }\end{array}$} & Predicted & 15.7 & $10.7,22.8$ & 21.8 & $9.2,51.8$ \\
\hline & Actual & 12.0 & $7.5,19.5$ & 16.0 & $0.3,743.5$ \\
\hline \multirow{2}{*}{$\begin{array}{l}\text { Smokers (not smoked on the day of } \\
\text { testing), atopic, asthmatic }\end{array}$} & Predicted & 22.8 & $13.6,36.9$ & 29.8 & $11.3,79.0$ \\
\hline & Actual & 24.0 & $17.8,32.4$ & 32.3 & $|4.6,7| .2$ \\
\hline \multirow{2}{*}{$\begin{array}{l}\text { Smokers (smoked on the day of testing), } \\
\text { non-atopic, non-asthmatic }\end{array}$} & Predicted & 7.0 & $5.6,8.8$ & 6.5 & $5.1,8.2$ \\
\hline & Actual & 7.3 & $6.4,8.3$ & 6.5 & $5.8,7.3$ \\
\hline \multirow{2}{*}{$\begin{array}{l}\text { Smokers (smoked on the day of testing), } \\
\text { atopic, non-asthmatic }\end{array}$} & Predicted & 10.1 & $7.2,14.0$ & 8.9 & $6.3,12.5$ \\
\hline & Actual & 10.2 & $8.8,11.8$ & 8.9 & $7.3,10.9$ \\
\hline \multirow{2}{*}{$\begin{array}{l}\text { Smokers (smoked on the day of testing), } \\
\text { non-atopic, asthmatic }\end{array}$} & Predicted & 8.7 & $6.0,12.5$ & 8.0 & $3.8,17.0$ \\
\hline & Actual & 8.9 & $7.3,10.9$ & 8.1 & $4.0,16.4$ \\
\hline \multirow{2}{*}{$\begin{array}{l}\text { Smokers (smoked on the day of testing), } \\
\text { atopic, asthmatic }\end{array}$} & Predicted & 12.6 & $7.6,20.3$ & 11.0 & $4.6,26.0$ \\
\hline & Actual & 9.3 & $6.2,13.8$ & 10.9 & $6.6,18.0$ \\
\hline
\end{tabular}

result. More recently, Olivieri et al. have reported higher levels in males, with an upper limit of normal of $28.8 \mathrm{ppb}$, compared to $21.5 \mathrm{ppb}$ for females [27]. Travers et al. [19] reported that the mean $\mathrm{F}_{\mathrm{E}} \mathrm{NO}$ in males was $23 \%$ higher than in females (95\% C.I. $7-43 ; \mathrm{p}=0.004, \mathrm{n}=191)$. In that study, the significance of the difference persisted even after controlling for height. In the study by Berry et al. a similar highly significant difference between males and females was recorded [28]. However, in the largest study to date to focus on factors affecting $\mathrm{F}_{\mathrm{E}} \mathrm{NO}$, comprising 2,200 subjects, Olin et al. has presented contrasting results [18]. Although there was a male-female $\mathrm{F}_{\mathrm{E}} \mathrm{NO}$ difference in non-smokers amounting to $19 \%$, this was not statistically significant in a multiple linear regression analysis in which adjustments for all other factors were included [18]. The reasons why the difference failed to reach statistical significance are unclear.
After adjusting for sex, we found that other anthropometric factors such as height and lung function were no longer significant factors affecting $\mathrm{F}_{\mathrm{E}} \mathrm{NO}$. Previously it has been argued that sex-related differences in $\mathrm{F}_{\mathrm{E}} \mathrm{NO}$ result from differences in the surface area of airway epithelium, the major source of exhaled NO, and for which height is an important anthropometric correlate. Thus our results are perhaps surprising. However, given that plasma levels of nitrate, a product of NO metabolism, are similarly different between the sexes $[25,29]$, it seems unlikely that NO production in the airways is solely a reflection of differences in airway size, but rather reflects sex-related differences in endogenous NO production. This is consistent with the results of a twin study, which showed that genetic rather than environmental factors are more important in determining $\mathrm{F}_{\mathrm{E}} \mathrm{NO}[30]$.

Our findings raise the question as to whether guidelines for the interpretation of $\mathrm{F}_{\mathrm{E}} \mathrm{NO}$ should be stratified by sex, 
and that reference ranges for males and females should be different. In the paper by Olivieri et al. the authors propose that reference ranges should be stratified for sex [27]. Travers et al. [19] advocate reference ranges based on sex, smoking status and atopy, but not age or height. We concur with this view, and the reference ranges contained in Table 5 of the present paper are based on this approach. In the study by Olin et al. [18], similar to conventional pulmonary function tests, both age and height but not sex, were deemed to be significant, although reference values as such were not provided. All studies concur that smoking and atopy are important considerations, and both are included in the reference values given here and by Travers et al. [19].

In fact, interpreting $\mathrm{F}_{\mathrm{E}} \mathrm{NO}$ levels in clinical practice is even more complex. Reference values which take into account background characteristics such as sex, atopy and smoking may indeed be useful in guiding the diagnosis of airwaysrelated symptoms. In asymptomatic individuals, it is still possible that increased $\mathrm{F}_{\mathrm{E}} \mathrm{NO}$ reflects subclinical airway inflammation $[7,28]$, but this interpretation is less likely if appropriate reference values which take factors such as sex into account have been used in the first place. The interpretation of $\mathrm{F}_{\mathrm{E}} \mathrm{NO}$ levels in the context of ongoing management of diagnosed asthma is far from clear. Despite optimal anti-inflammatory treatment, $\mathrm{F}_{\mathrm{E}} \mathrm{NO}$ levels may remain resolutely high [31], and it is generally agreed that normalizing $\mathrm{F}_{\mathrm{E}} \mathrm{NO}$ in relation to reference values for a healthy population is not a desirable therapeutic aim [8]. This point is perhaps reflected in the results obtained in the present study, which showed that in nonsmoking, atopic, male asthmatics, who were all clinically stable, the upper limit of the 95\% confidence interval was $38.8 \mathrm{ppb}$, considerably higher than the levels obtained in non-smoking, atopic, male, non-asthmatics (28.2 ppb).

One of the weaknesses of our study is that the $\mathrm{F}_{\mathrm{E}} \mathrm{NO}$ measurements were obtained in individuals who were all aged 32 years. Thus it was not possible to explore the influence of age as a factor in the regression analyses or to conclude whether reference values ought to include it as a factor. Previous studies have reported that $\mathrm{F}_{\mathrm{E}} \mathrm{NO}$ rises with increasing age in children $[15,16,32-34]$. In adults, Olin et al. [18] have reported that an effect of age also occurs: $\mathrm{F}_{\mathrm{E}} \mathrm{NO}$ was shown to increase over the age range 35 to 65 years, with the magnitude of effect similar to that of atopy. In contrast, in the study by Travers et al. no significant relationship was noted over a similar age range, but numbers were much smaller [19]. In children, it is suggested that the changes with age are attributable to increasing airway NO flux, probably reflecting larger airway surface area with growth [34]. If at all, any increase in $\mathrm{F}_{\mathrm{E}} \mathrm{NO}$ with age in adults is likely to be due to non-anthropometric factors, and if the results from Olin et al. are repeatable, this may be an important consideration.

In summary, our data confirm that differences in $\mathrm{F}_{\mathrm{E}} \mathrm{NO}$ between males and females are of sufficient magnitude that the interpretation of $\mathrm{F}_{\mathrm{E}} \mathrm{NO}$ should be stratified by sex. This approach should be incorporated into clinical practice. Other common and easily identified factors such as current smoking and atopy also require to be taken into account when interpreting $\mathrm{F}_{\mathrm{E}} \mathrm{NO}$ values in adults. Contrasting results from a number of studies still leave open the question as to whether age and height ought to be included in future reference equations. These outstanding issues add to the current challenges which still remain in the application and interpretation of $\mathrm{F}_{\mathrm{E}} \mathrm{NO}$ levels in clinical practice, and require further study.

\author{
Abbreviations \\ C.I. confidence interval \\ $\mathrm{F}_{\mathrm{E}} \mathrm{NO}$ fraction of nitric oxide in expired air \\ $\mathrm{FEV}_{1}$ forced expiratory volume in one second \\ FVC forced vital capacity \\ NO nitric oxide \\ $\mathrm{mL} / \mathrm{sec}$ milliliters per second \\ ppb parts per billion \\ SPT skin prick test
}

\section{Competing interests}

D Robin Taylor has received lecture fees and an unrestricted educational grant valued at $\$ 20,000$, from Aerocrine, a manufacturer of nitric oxide analysers

All other named authors have no competing interests to declare in relation to the contents of this manuscript.

\section{Authors' contributions \\ D Robin Taylor devised the study plan and wrote the manuscript}

Piush Mandhane conducted statistical analysis of the results and contributed to writing the manuscript

Justina M Greene conducted statistical analysis of the results

Robert J. Hancox directed and supervised the conduct of the study and data collection 
Christene R McLachlan provided technical assistance in the collection of study data

Avis J Williamson provided technical assistance in the collection of study data

Sue Filsell provided technical assistance in the collection of study data

Jan O Cowan provided technical assistance in the collection of study data

Andrew D Smith provided technical assistance in the collection of study data

Malcolm R Sears provided general study oversight and contributed to writing the manuscript

All named authors have approved the contents of this manuscript.

\section{Additional material}

\section{Additional File 1}

Selection of adjusted linear regression models for all study members, and stratified by males and females

Click here for file

[http://www.biomedcentral.com/content/supplementary/1465-

9921-8-82-S1.doc]

\section{Acknowledgements}

The authors wish to acknowledge the loyalty of each of the Study members of the Dunedin Multidisciplinary Health and Development Study, and the support of Michelle McCann and Professor Richie Poulton. Professor Temi Moffitt kindly permitted the use of data pertaining to cannabis smoking.

\section{References}

I. Smith AD, Taylor DR: Is exhaled nitric oxide measurement a useful clinical test in asthma? Curr Opin Allergy Clin Immunol 2005, 5:49-56.

2. Jatakanon A, Lim S, Kharitonov SA, Chung KF, Barnes PJ: Correlation between exhaled nitric oxide, sputum eosinophils, and methacholine responsiveness in patients with mild asthma. Thorax 1998, 53:91-5.

3. Berlyne GS, Parameswaran K, Kamada D, Efthimiadis A, Hargreave FE: A comparison of exhaled nitric oxide and induced sputum as markers of airway inflammation. J Allergy Clin Immunol 2000, 106:638-44.

4. Warke TJ, Fitch PS, Brown V, Taylor R, Lyons JD, Ennis M, Shields MD: Exhaled nitric oxide correlates with airway eosinophils in childhood asthma. Thorax 2002, 57:383-7.

5. Brightling CE, Symon FA, Birring SS, Bradding P, Wardlaw AJ, Pavord ID: Comparison of airway immunopathology of eosinophilic bronchitis and asthma. Thorax 2003, 58:528-32.

6. Payne DN, Adcock IM, Wilson NM, Oates T, Scallan M, Bush A: Relationship between exhaled nitric oxide and mucosal eosinophilic inflammation in children with difficult asthma, after treatment with oral prednisolone. Am J Respir Crit Care Med 200I, 164:1376-8I.

7. van den Toorn LM, Overbeek SE, Overbeek SE, de Jongste JC, Leman $\mathrm{K}$, Hoogsteden HC, Prins JB: Airway inflammation is present during clinical remission of atopic asthma. Am J Respir Crit Care Med 200I, 164:2107-13.

8. Taylor DR, Pijnenburg MW, Smith AD, De Jongste JC: Exhaled nitric oxide measurements: clinical application and interpretation. Thorax 2006, 61:817-27.

9. Kharitonov SA, Robbins RA, Yates D, Keatings V, Barnes PJ: Acute and chronic effects of cigarette smoking on exhaled nitric oxide. Am J Respir Crit Care Med 1995, 152:609-12.

10. Persson MG, Zetterstrom O, Agrenius V, Ihre E, Gustafsson LE: Single-breath nitric oxide measurements in asthmatic patients and smokers. Lancet 1994, 343:146-7.

II. van Amsterdam JG, Janssen NA, de Meer G, Fischer PH, Nierkens S, van Loveren H, Opperhuizen A, Steerenberg PA, Brunekreef B: The relationship between exhaled nitric oxide and allergic sensitization in a random sample of school children. Clin Exp Allergy 2003, 33:|87-9|.

12. Jouaville LF, Annesi-Maesano I, Nguyen LT, Bocage AS, Bedu M, Caillaud D: Interrelationships among asthma, atopy, rhinitis and exhaled nitric oxide in a population-based sample of children. Clin Exp Allergy 2003, 33:1506-II.

13. Gratziou C, Lignos M, Dassiou M, Roussos C: Influence of atopy on exhaled nitric oxide in patients with stable asthma and rhinitis. Eur Respir J 1999, 14:897-901.

14. Henriksen AH, Sue-Chu M, Lingaas Holmen T, Langhammer A, Bjermer $L$ : Exhaled and nasal NO levels in allergic rhinitis: relation to sensitization, pollen season and bronchial hyperresponsiveness. Eur Respir J 1999, 13:30 I-6.

15. Buchvald F, Baraldi E, Carraro S, Gaston B, De Jongste J, Pijnenburg $M W$, Silkoff $P E$, Bisgaard $\mathrm{H}$ : Measurements of exhaled nitric oxide in healthy subjects age 4 to 17 years. J Allergy Clin Immunol 2005, I I 5: I 130-6.

16. Avital A, Uwyyed K, Berkman N, Bar-Yishay E, Godfrey S, Springer C: Exhaled nitric oxide is age-dependent in asthma. Pediatr Pulmonol 2003, 36:433-8.

17. Cardinale F, de Benedictis FM, Muggeo V, Giordano P, Loffredo MS, lacoviello G, Armenio L: Exhaled nitric oxide, total serum IgE and allergic sensitization in childhood asthma and allergic rhinitis. Pediatr Allergy Immunol 2005, 16:236-42.

18. Olin AC, Rosengren A, Thelle DS, Lissner L, Bake B, Toren K: Height, age, and atopy are associated with fraction of exhaled nitric oxide in a large adult general population sample. Chest 2006, 130:1319-25.

19. MS Travers J, S Aldington, M Williams, P Shirtcliffe, A Pritchard, M Weatherall, $R$ Beasley: Reference ranges for exhaled nitric oxide derived from a random community survey of adults. American Journal of Respiratory and Critical Care Medicine 2007.

20. Sears MR, Greene JM, Willan AR, Wiecek EM, Taylor DR, Flannery EM, Cowan JO, Herbison GP, Silva PA, Poulton R: A longitudinal, population-based, cohort study of childhood asthma followed to adulthood. N Engl J Med 2003, 349:|4|4-22.

21. SW Silva PA: From child to adult: The Dunedin Multidisciplinary Health and Development Study Auckland: Oxford University Press; 1996.

22. ATS/ERS Recommendations for Standardized Procedures for the Online and Offline Measurement of Exhaled Lower Respiratory Nitric Oxide and Nasal Nitric Oxide 2005. Am J Respir Crit Care Med 2005, 171:912-930.

23. Smith AD, Cowan JO, Brassett KP, Herbison GP, Taylor DR: Use of exhaled nitric oxide measurements to guide treatment in chronic asthma. N Engl J Med 2005, 352:2163-73.

24. Kharitonov SA, Gonio F, Kelly C, Meah S, Barnes PJ: Reproducibility of exhaled nitric oxide measurements in healthy and asthmatic adults and children. Eur Respir J 2003, $21: 433-8$.

25. Jilma B, Kastner J, Mensik C, Vondrovec B, Hildebrandt J, Krejcy K, Wagner OF, Eichler HG: Sex differences in concentrations of exhaled nitric oxide and plasma nitrate. Life Sci 1996, 58:469-76.

26. Tsang KW, Ip SK, Leung R, Tipoe GL, Chan SL, Shum IH, Ip MS, Yan $C$, Fung PC, Chan-Yeung M, et al.: Exhaled nitric oxide: the effects of age, gender and body size. Lung 200I, 179:83-91.

27. Olivieri M, Talamini G, Corradi M, Perbellini L, Mutti A, Tantucci C, Malerba M: Reference values for exhaled nitric oxide (reveno) study. Respir Res 2006, 7:94.

28. Berry MA, Shaw DE, Green RH, Brightling CE, Wardlaw AJ, Pavord ID: The use of exhaled nitric oxide concentration to identify eosinophilic airway inflammation: an observational study in adults with asthma. Clin Exp Allergy 2005, 35: I I75-9. 
29. Takahashi $H$, Nakanishi T, Nishimura M, Tanaka H, Yoshimura $M$ : Measurements of serum levels of nitrate ions in men and women: implications of endothelium-derived relaxing factor in blood pressure regulation and atherosclerosis. J Cardiovasc Pharmacol 1992, 20:S214-6.

30. Lund MB, Kongerud J, Nystad W, Boe J, Harris JR: Genetic and environmental effects on exhaled nitric oxide and airway responsiveness in a population-based sample of twins. Eur Respir J 2006, I:I.

31. Pijnenburg MW, Bakker EM, Lever S, Hop WC, De Jongste JC: High fractional concentration of nitric oxide in exhaled air despite steroid treatment in asthmatic children. Clin Exp Allergy 2005, 35:920-5.

32. Kissoon N, Duckworth LJ, Blake KV, Murphy SP, Taylor CL, DeNicola LR, Silkoff PE: Exhaled nitric oxide concentrations: online versus offline values in healthy children. Pediatr Pulmonol 2002, 33:283-92.

33. Franklin PJ, Taplin R, Stick SM: A community study of exhaled nitric oxide in healthy children. Am J Respir Crit Care Med 1999, 159:69-73.

34. Latzin P, Beck J, Griese M: Exhaled nitric oxide in healthy children: variability and a lack of correlation with atopy. Pediatr Allergy Immunol 2002, 13:37-46.

Publish with Biomed Central and every scientist can read your work free of charge

"BioMed Central will be the most significant development for disseminating the results of biomedical research in our lifetime. "

Sir Paul Nurse, Cancer Research UK

Your research papers will be:

- available free of charge to the entire biomedical community

- peer reviewed and published immediately upon acceptance

- cited in PubMed and archived on PubMed Central

- yours - you keep the copyright

Submit your manuscript here:

http://www.biomedcentral.com/info/publishing_adv.asp 